\title{
GERENCIAMENTO INTEGRADO DE RESÍDUOS SÓLIDOS E SUA APLICABILIDADE EM PRODUÇÃO DE REFEIÇÕES: UM DIÁLOGO INTERDISCIPLINAR
}

\author{
C. L. CARNEIRO \\ Universidade Federal do Rio Grande do Norte \\ claudialeite@supercabo.com.br
}

Artigo submetido em julho/2013 e aceito em fevereiro/2014

\section{RESUMO}

O gerenciamento integrado de resíduos sólidos ajustado a normas específicas dos Serviços de Alimentação e Nutrição torna-se uma ferramenta operacional para melhoria na gestão ambiental. Nesta pesquisa, os resíduos produzidos foram caracterizados e classificados de acordo com sua natureza. Como resultado da aplicação do plano, observou-se um expressivo volume de resíduos gerados, uma cultura de desperdício, que agride o meio ambiente e reflete nos custos e gastos de um restaurante universitário. É enfatizada a importância da promoção de ações que permitam a aplicação do Gerenciamento Integrado de Resíduos Sólidos para que se alcancem efeitos positivos na instituição nas áreas econômica e ambiental.

PALAVRAS-CHAVE: Resíduos Sólidos, Unidade de Alimentação e Nutrição, Plano de Gerenciamento Integrado de Resíduos Sólidos.

\section{INTEGRATED MANAGEMENT OF SOLID WASTE AND THE APPLICABILITY IN PRODUCTION OF MEALS: AN INTERDISCIPLINARY DIALOGUE}

\begin{abstract}
The integrated management of solid waste set according to specific standards of the Food and Nutrition Services becomes an operational tool for improved environmental management. In this research, the waste produced were characterized and classified according to their nature. As a result of the implementation of the plan, a significant volume of generated waste was
\end{abstract}

observed, a culture of waste that harms the environment and reflects itself in the costs and expenses of a university restaurant. It is emphasized the importance of promoting actions that enable the application of this integrated management of solid waste in order to achieve positive effects in the institution in the economic and environmental areas.

KEYWORDS: Solid Waste, Food and Nutrition Unit, Integrated Management Plan for Solid Waste. 


\section{INTRODUÇÃO}

O gerenciamento integrado de resíduos sólidos (GIRS) é definido atualmente pela legislação que institui a Política Nacional de Resíduos Sólidos, na Lei 12.305/2010, como um "conjunto de ações exercidas direta ou indiretamente, nas etapas de coleta, transporte, transbordo, tratamento e destinação final ambientalmente adequada dos resíduos gerados e disposição final ambientalmente adequada dos rejeitos, de acordo com plano municipal de gestão integrada de resíduos sólidos ou com plano de gerenciamento sólidos exigidos nesta Lei" (BRASIL, 2010).

Para autores que atuam na área, a coleta, tratamento e disposição inadequada de resíduos sólidos têm fortes impactos sociais e econômicos. Entretanto, apesar de muitos desses impactos negativos serem de amplo conhecimento, a questão dos resíduos tem sido, por muitas vezes, negligenciada, produzindo situações potencialmente destrutivas e refletindo esses danos sobre gerações futuras (MENEZES e MENEZES, 1999).

Dentre os variados sistemas produtivos geradores de resíduos, destacam-se as Unidades de Alimentação e Nutrição (UAN). Na realidade operacional da produção de refeições, isto acontece desde, o recebimento dos gêneros alimentícios, as etapas de pré-preparo, de preparo e até a distribuição final.

Compreender a dinâmica da geração destes resíduos, com objetivo de preservar o meio ambiente, é fundamental para que haja interação da implantação do plano de gerenciamento de resíduos com as rotinas de produção de uma Unidade de Alimentação e Nutrição e permita-se a abertura para um diálogo interdisciplinar.

O diálogo de instrumentos da gestão ambiental, como por exemplo, o plano de Gerenciamento Integrado de Resíduos Sólidos e as etapas da produção de refeições em Unidades de Alimentação e Nutrição, podem gerar dificuldades particulares a interdisciplinaridade. Entretanto, o dilema ambiental contemporâneo faz valer a necessidade urgente dessa premissa.

Além da produção de refeições com qualidade nutricional e sensorial, os gestores e os técnicos envolvidos na produção em UANs deveriam fazer uso de práticas e processos de trabalho ambientalmente corretos.

Infere-se que a conservação ambiental é uma questão a ser enfrentada e defendida, e, assim sendo, os sistemas de produção, sejam estes de amplitude a quaisquer finalidades propostas, os gestores dos referidos sistemas necessitam conscientizar-se que, no decorrer de seus processos produtivos, se houver uma condução irresponsável, esta postura agredirá cada vez mais o meio ambiente. É neste foco que se propõe a construção do entendimento de conceitos e etapas que facilitarão a implantação de um plano Integrado de Gerenciamento de Resíduos Sólidos e o diálogo interdisciplinar com a Ciência da Nutrição, em produção de refeições.

\section{MÉTODO DA PESQUISA}

O método da pesquisa empregado foi a pesquisa-ação. A análise envolveu um restaurante institucional, incluindo investigação das etapas do sistema de produção "in loco". 
O atendimento do restaurante no período da coleta compreendeu 2000 refeições/dia aproximadamente, sendo dispostas em desjejuns, almoços e jantares.

A análise observacional identificou um grande número de resíduos gerados, e assim levantaram-se dados e postulados entendimentos sobre o processo produtivo. Com a interação cooperativa entre a pesquisa e os participantes atuantes (gestor, funcionários e bolsistas) na situação observada.

Metas foram formuladas para a aplicação de um plano de Gerenciamento de Resíduos Sólidos. Aplicou-se um questionário com perguntas objetivas aos funcionários e à chefia, levantando questões sobre achados pessoais, percepção do resíduo gerado e vias de como minimizar e melhorar a produção dos resíduos.

A coleta de dados foi realizada em vinte um dias consecutivos, incluindo os fins de semana. A amostragem diária foi coletada visando pesagem de todos os resíduos gerados por dia, no período da coleta e os registros foram anotados em planilha simples, contendo informações sobre a data, tipo de resíduo gerado, peso e local de origem. Depois, foram somados os quantitativos das amostras diárias durante os 21 dias.

Os custos diretos de produção dos gêneros alimentícios desperdiçados foram margeados considerando o índice de Resto (IR) segundo Teixeira (1997) onde se divide o peso da refeição rejeitada, pela refeição distribuída resultando no número de refeições desperdiçadas. Para analisar o valor dos custos de produção em gêneros alimentícios multiplicou-se o valor encontrado de número de refeições desperdiçadas pelo preço de venda por refeição em reais nos 21 dias de análise

Para aplicação do plano, foram caracterizados os resíduos produzidos, partindo de um levantamento do quantitativo e qualitativo dos resíduos totais do local, para obter o percentual de resíduo úmido (orgânico) e resíduo seco (inorgânico) gerado. Em sequência, foi calculada a média diária de produção do resíduo úmido e seco, separadamente.

O método escolhido, de acordo com a disposição da sua natureza (orgânica ou inorgânica) foi segundo classificação da NBR ISO 14004 (1996): Sistemas de gestão ambiental: com diretrizes gerais sobre princípios, sistemas e técnicas de apoio, adaptada por Kinasz (2007) que ajusta as normas à realidade específica dos serviços de alimentação e nutrição, como descritos abaixo:

- PAPEL/PAPELÃO: caixas de papelão, sacos e sacolas de papel, outros tipos de invólucros de papel ou papelão, toalhas e guardanapos de papel;

- VIDROS: vidros de acondicionamento de alimentos e de produtos de higiene;

- LATAS: latas de acondicionamento de alimentos de produtos de higiene e desinfecção;

- APARAS E RESÍDUOS DE PRÉ-PREPARO E PREPARO DE ALIMENTOS: partes retiradas dos alimentos como cascas, sementes, talos, folhas danificadas, raízes, pele, cartilagens, ossos, entre outras, nas atividades denominadas de pré-preparo (fase que envolve a lavagem, limpeza, corte e temperos dos alimentos a serem preparados). Preparo dos alimentos e restos de alimentos contidos nos utensílios.

- RESTOS/SOBRAS: restos das preparações que compõem o cardápio e que são deixados pelo cliente na bandeja/prato. As sobras são: preparações não consumidas pelos clientes e que não são passíveis de reutilização. 
O modelo escolhido para a elaboração do Plano de Gerenciamento foi o modelo proposto por Menezes e Menezes (1999), tendo como critério de seleção a característica de ser organizacional e didaticamente similar ao modelo organizacional do Manual de Boas Práticas de Fabricação (BRASIL, 2004), que é um instrumento utilizado obrigatoriamente na Nutrição, o que poderá facilitar a elaboração e os entendimentos interdisciplinares.

Na descrição, o plano consistiu em três etapas bem definidas: Etapa 1 - Identificação do gerador, com dados descritivos da UAN ; Etapa 2 - Diagnóstico da situação atual e dos Resíduos gerados segundo a classificação de Kinasz (2007); Etapa 3 - com a Descrição do Plano de Gerenciamento Aplicado na unidade.

\section{RESULTADOS E DISCUSSÃO}

Os participantes da pesquisa entrevistados foram $94 \%$ do sexo masculino (94\%) e $74 \%$ com idades entre 22 e 40 anos; possuem menos de 5 anos de tempo de serviço; e $44 \%$ com grau de escolaridade com ensino médio completo. Consolidando um perfil masculino, pouco tempo de trabalho; idade mediana jovem e médio grau de instrução.

Quanto à questão das maiores dificuldades para minimizar os problemas dos resíduos, os funcionários pesquisados consideram que as maiores dificuldades para minimizar os problemas dos resíduos são: a conscientização (16\%), a prática operacional de separação dos resíduos (9\%), Educação (9\%) e disposição (9\%).

Este modelo de referência proposto por Menezes e Menezes (1999) tem sua conformação bastante similar ao Manual de Boas Práticas de Fabricação, descrito pela Resolução RDC №. 216 (BRASIL, 2004), tornando-o extremante conveniente, de fácil replicação, exequível e oportuno na elaboração de um plano de gerenciamento integrado de resíduos sólidos em restaurantes. A descrição em três etapas facilitou a elaboração do plano de gerenciamento ajustado à unidade em questão.

$\mathrm{Na}$ aplicação do Plano na pesquisa, na etapa I de Identificação do gerador, obtiveram-se informações gerais quanto à tipologia do empreendimento: descrição da atividade, fluxograma e os procedimentos realizados no empreendimento; a origem dos recursos destinados ao financiamento. A Unidade encontra-se representada literal e graficamente segundo o Estatuto da instituição a que é vinculada, sendo descritos ainda nesta etapa a razão social, o cadastro nacional de pessoa jurídica, nome fantasia, tipo de serviços e fluxogramas de operações. Permitindo a visualização do sistema organizacional.

Na etapa II do Plano - Diagnóstico da situação atual e dos Resíduos gerados, identificaramse dados para a reavaliação dos sistemas produtivos e operacionais que contribuem para ajustes globais nas unidades de alimentação e que são moldados pela sustentabilidade. Os resultados para a caracterização, segundo a natureza e de acordo com classificação de Kinacz (2007), estão demonstrados na Tabela 1: 
Tabela 1 - Caracterização segundo à natureza baseada na classificação de Kinacz (2007). Natal, Maio /2011.

\begin{tabular}{c|c|c}
\hline RESÍDUOS & TOTAL/kg & VALOR RELATIVO (\%) \\
\hline Papel/papelão & 24,73 & 0,2 \\
\hline Vidros & 13,69 & 0,1 \\
\hline Latas & 316,05 & 2,8 \\
\hline Resíduos e aparas/ preparo & $6.375,57$ & 56,2 \\
\hline Restos e sobras & $3.599,33$ & 31,8 \\
\hline Outros componentes & $1.012,06$ & 8,9 \\
\hline TOTAL & $11.341,33$ & $100 \%$ \\
\hline
\end{tabular}

Para cerca de 9.974,90 kg de resíduos úmido (orgânico) e 1.366,43 kg de seco (inorgânico), observaram-se os per capitas diários de $0,22 \mathrm{~kg} /$ comensal/dia e 0,03 $\mathrm{kg} /$ comensal/dia, respectivamente. Com maioria de $87 \%$ dos resíduos gerados serem úmidos (orgânicos). Metas de redução dos resíduos orgânicos produzidos devem ser analisadas criteriosamente com a monitoração adequada de cortes, aparas, modo de preparo e recebimento de gêneros na cozinha, com a finalidade de haver uma produção mais limpa, bem como redução dos custos. Margeando os custos diretos de produção foi desperdiçado em gêneros alimentícios um total de 18.381,86 reais para os 21 dias de análise. Estes custos foram margeados como descrito no método da pesquisa.

Os principais pontos de desperdícios identificados foram em três setores: a produção (cozinha) com 75\%, a devolução (copa de bandejas e pratos) com $22 \%$ de resíduos gerados e almoxarifado com $3 \%$.

Os valores encontrados demonstram a importância da prevenção, pois como cita Teixeira (1997), ela permite o desenvolvimento e implantação de um sistema de análise e apresentação de dados, conhecimento e aplicabilidade de fluxograma de processos, bem como um plano de controle de qualidade das atividades. O que reforça a importância do diagnóstico operacional e produtivo para a execução do plano de gerenciamento.

Na etapa III, ocorreu a descrição do plano de gerenciamento aplicado na unidade. Com dados da instituição e do órgão de alimentação, classificação dos resíduos gerados e orientações norteadoras para coleta, segregação, transporte e destinação final, com contatos (telefônicos/email) de associações de catadores e empresas conveniadas a instituição.

Quanto à destinação dos resíduos passíveis de reutilização ou reciclagem, foram fornecidos nome da empresa geradora e das conveniadas para reciclagem, com endereço, telefone/fax e dados do responsável técnico, assim como os procedimentos de manejo utilizados na segregação dos resíduos. Definidas e descritas formas de acondicionamento correto, baseadas na conformidade com a RDC 216/2004 que trata das boas práticas de produção de refeições, por tipo ou grupo de resíduos, e os tipos de recipientes utilizados para o acondicionamento foram descritos conforme a capacidade e estabelecidos procedimentos para o correto fechamento, vedação e manuseio dos recipientes, de forma a evitar vazamentos e/ou ruptura dos mesmos e, inseridos símbolos de identificação compatível com o tipo de resíduo acondicionado.

Para a coleta e transporte interno dos resíduos foram descritos os procedimentos das medidas a serem adotadas em caso de rompimento de recipientes, vazamento de líquidos, derrame de resíduos, ou ocorrência de outras situações indesejáveis, bem como a forma de 
higienização dos recipientes e equipamentos e os produtos empregados, apresentando planta baixa do estabelecimento e especificando as rotas dos resíduos. Descrita ainda a área de armazenamento temporário, obedecendo às medidas de segurança e proteção ambiental de impermeabilização do piso; cobertura e ventilação; isolamento e sinalização; acondicionamento adequado; controle de operação; treinamento de pessoal e monitoramento da área.

$\mathrm{Na}$ coleta e transporte externo para aterro sanitário, foram especificados os resíduos por grupo, a frequência, horário e tipo de veículo transportador, e indicada à empresa responsável pela coleta externa, fornecendo nome, endereço, telefone/fax e os dados do responsável técnico.

O reconhecimento das operações produtivas associadas à aplicabilidade e a implantação de um plano de gerenciamento integrado de resíduos permitem a promoção de ajustes amplos nas instituições, nas quais vários atores certamente devem contribuir para que metas comuns sejam alcançadas, sendo passo importante para entendimentos reais de modificação cultural, onde conceitos de segurança ambiental global e de desenvolvimento são centrais para o estabelecimento de técnicas de manejo ambiental, de combate ao desperdício e à poluição (RIBEIRO, 2005).

As demandas de responsabilidade ambiental passam por associações produtivas e também pela sociedade como um todo. Assim sendo, as unidades produtoras de refeições não devem e nem podem se eximir desta realidade. De modo abrangente, poder-se-ia afirmar que o oferecimento de uma alimentação ambientalmente equilibrada constitui um instrumento sincrônico de preparações nutricionalmente adequadas, com custos equacionados com a realidade da empresa e, ainda assim, com produção consciente e respeitosa em relação ao meio ambiente. Facilitando o diálogo interdisciplinar entre a gestão ambiental e a produção de refeições.

A revisão dos processos produtivos em UAN, estabelece objetivos ambientais, integram organogramas, fluxogramas e permite a redação de Planos de Gerenciamentos de Resíduos Integrados. Assim, poder-se-á estabelecer os sistemas de controle das operações, de gestões e de registros nessas referidas unidades, dialogando-se interdisciplinarmente com mais eficácia.

\section{CONSIDERAÇÕES FINAIS}

O plano de Gerenciamento Integrado de Resíduos Sólidos tem como finalidade estabelecer orientações para aperfeiçoar as operações de manejo de resíduos. Sua interlocução com a manipulação de alimentos expressa o objetivo de promover o gerenciamento integrado de resíduos sólidos, definindo um padrão de qualidade e identidade dos insumos e dos produtos gerados e a sua operacionalização ambientalmente correta.

Em função do expressivo volume de resíduos produzidos na UAN, pode-se afirmar que existe uma cultura de desperdício, onde o ato de produção, consumo e disposição de alimentos está sendo gerado além do que é socialmente necessário ou ambientalmente sustentável, contribuindo assim para o aumento da geração de resíduos sólidos de produtos e de alimentos. $O$ novo cenário ambiental em construção busca um gradativo abandono do modelo linear de produção vigente, no qual a capacidade de exploração das fontes de materiais e energia, com aumento do consumo de bens, explora ao máximo a capacidade de saturação do ambiente. Este 
aspecto demonstra a irresponsabilidade com que nossa espécie tem tratado a questão dos recursos naturais, ocasionando exploração das matérias primas, na maioria das vezes, além da capacidade de regeneração destas. Assim, numa época de aumento da consciência ambiental, urge a necessidade do crescente conhecimento das implicações sobre o meio ambiente, a responsabilidade e cuidado com os subprodutos dos sistemas produtivos.

A perspectiva da sustentabilidade deve ser incorporada ao gerenciamento desses resíduos gerados pelas instituições como um todo, ressaltando-se a importância da elaboração de um Plano de Gerenciamento Integrado de Resíduos em UANS, que atua como ferramenta metodológica para reiterar a importância do diálogo interdisciplinar das Ciências que tratam as questões Ambientais e a Nutrição.

\section{REFERÊNCIAS BIBLIOGRÁFICAS}

1. ASSOCIAÇÃO BRASILEIRA DE NORMAS TÉCNICAS. NBR ISO 14004: Sistemas de gestão ambiental: diretrizes gerais sobre princípios, sistemas e técnicas de apoio. Rio de Janeiro, 1996a.

2. BRASIL. Agência Nacional de Vigilância Sanitária. Lei № 12.305 de 02 de Agosto de 2010. Institui a Política Nacional de Resíduos Sólidos; altera a Lei no 9.605, de 12 de fevereiro de 1998; e dá outras providências. Disponível em: < http:// www.planalto.gov.br/ccivil_03/_ato2007-2010/lei/11230> Acesso: julho/2010.

3. BRASIL. Agência Nacional de Vigilância Sanitária. Resolução RDC no 216 de 15 de setembro de 2004. Dispõe sobre o regulamento Técnico de Boas Práticas para o Serviço de Boas Práticas de Alimentação. Diário Oficial da União, Brasília, DF, de 16 de setembro de 2004. Disponível em: <http://elegis.anvisa.gov.br/leisref/public/showAct.php?id=12546> Acesso em: 04 de set, 2009.

4. KINASZ, Tânia Regina. Reflexão Teórica sobre Gerenciamento de Resíduos Sólidos em Unidade de Alimentação e Nutrição. Revista Nutrição em Pauta, São Paulo, v. 15, p. 56-60, Nov/Dez. 2007.

5. MENEZES, Ricardo Amaral; MENEZES, Marco Amaral. Considerações sobre o Gerenciamento Integrado de Resíduos Sólidos (GIRS). 53. ed. p. 12-17. Out. 1999.

6. RIBEIRO, Lauro André L L. Avaliação de Barreiras para implementação de um sistema de Gestão Ambiental na UFRGS. In: XXV ENEGEP. Porto Alegre, RS, 29 Out. a 01 Nov., 2005.

7. TEIXEIRA, Suzana Maria Ferreira Gomes L L. Funcionamento das Unidades de Alimentação e Nutrição. In: . Administração aplicada às Unidades de Alimentação e Nutrição. p. 165219. São Paulo: Atheneu, 1997. 